\title{
ON THE INERTIA CONJECTURE FOR ALTERNATING GROUP COVERS
}

\author{
SOUMYADIP DAS AND MANISH KUMAR
}

\begin{abstract}
Aвstract. The wild part of Abhyankar's Inertia Conjecture for a product of certain Alternating groups is shown for any algebraically closed field of odd characteristic. For $d$ a multiple of the characteristic of the base field, a new étale $A_{d}$-cover of the affine line is obtained using an explicit equation and it is shown that it has the minimal possible upper jump.
\end{abstract}

\section{INTRODUCTION}

Let $k$ be an algebraically closed field of characteristic $p>0$ and let $G$ be a finite group. The quasi $p$-subgroup $p(G)$ is the subgroup generated by all the Sylow $p$-subgroups of $G$. Let $\phi: Y \rightarrow \mathbb{P}_{k}^{1}$ be a connected $G$-Galois cover of the projective line branched only at $\infty$. Then the induced $G / p(G)$-Galois cover is a prime-to- $p$ Galois cover of $\mathbb{P}^{1}$ branched only at $\infty$ and hence it is trivial. So $G=p(G)$. Such a group is called a quasi $p$-group. Abhyankar's 1957 conjecture ([1]) on the affine $k$-curves implies that a finite group $G$ occurs as the Galois group of a cover of the projective line branched only at $\infty$ if and only if $G$ is a quasi $p$-group. This conjecture for the affine line was proved by Serre ( $G$ solvable, [20]) and Raynaud ([17]).

Abhyankar in his 2001 paper ([3, Section 16]) conjectured a more refined statement on the Galois étale covers of the affine line which is known as the inertia conjecture. Let $G$ be a quasi $p$-group and $\phi: Y \rightarrow \mathbb{P}^{1}$ be a $G$-Galois cover of the projective line branched only at infinity. Then for any point $y$ of $Y$ in the fibre $\phi^{-1}(\infty)$, the inertia group $I$ at $y$ is an extension of a cyclic prime-to- $p$ group by a $p$-group $P$ ([19, Chapter IV, Corollary 4]). Let $J$ be the subgroup of $G$ generated by all the conjugates of $P$ in $G$. Then $J$ is a normal subgroup of $G$ and $Y \rightarrow \mathbb{P}^{1}$ induces a $G / J$-Galois cover of $\mathbb{P}^{1}$ which is at most tamely ramified at $\infty$. Since the tame fundamental group of $\mathbb{A}^{1}$ is trivial, $G=J$. Abhyankar's inertia conjecture says that the converse also holds.

The inertia conjecture can be compartmentalized into two parts (cf. [8, Conjecture 4.2]).

Conjecture 1.1. Let $G$ be a finite quasi p-group.

Wild Part: A p-subgroup P of G occurs as the inertia group of a G-Galois cover of $\mathbb{P}^{1}$ branched only at $\infty$ if and only if the conjugates of $P$ generate $G$.

Tame Part: Assume that a p-subgroup $P$ of $G$ occurs as the inertia group of a $G$ Galois étale cover of the affine line. Let $\beta$ be an element of $G$ of prime-to- $p$ order contained in the normalizer of $P$ in $G$. Let I be an extension of $\langle\beta\rangle$ by $P$ in $G$. Then there is a G-Galois cover $\phi: Y \rightarrow \mathbb{P}^{1}$ branched only at $\infty$ with inertia group I at a point in $Y$ over $\infty$.

By Abhyankar's Lemma ([18, XIII, Proposition 5.2]), one can reduce the tame part of the inertia group. On the other hand, Harbater has shown ([6, Theorem 2]) that the wild part of the inertia group can be increased. As a consequence, the inertia conjecture

Key words and phrases. Galois Cover, Inertia Conjecture, Ramification. 
is known for Sylow $p$-subgroups of any quasi $p$-group. In [13] it was shown that under certain conditions on the ramification filtration of the inertia group, its wild part can be made a little smaller.

The conjecture in general is wide open though some results supporting the conjecture have been obtained (see [4], [15], [14], [12] and [8] for more details).

For a quasi $p$-group $G$ and a subgroup $I$, we say that the pair $(G, I)$ is realizable if there exists a $G$-Galois cover $Y \rightarrow \mathbb{P}^{1}$ étale away from $\infty$ such that the inertia group at a point in $Y$ above $\infty$ is $I$. In this paper we first show that $\left(A_{d}, P\right)$ is realizable where $A_{d}$ is an Alternating group and $P$ is a $p$-subgroup containing a $p$-cycle (Corollary 4.7). Let $G_{1}$ and $G_{2}$ be perfect quasi $p$-groups, $I_{1}$ be a $p$-cyclic subgroup of $G_{1}$ and $I_{2}$ a cyclic $p$-subgroup of $G_{2}$. We show that if $\left(G_{1}, I_{1}\right)$ and $\left(G_{2}, I_{2}\right)$ are realizable then $\left(G_{1} \times G_{2}, I\right)$ is realizable for some cyclic $p$-subgroup $I$ of $G_{1} \times G_{2}$ such that its image in $G_{1}$ and $G_{2}$ are $I_{1}$ and $I_{2}$ respectively (Theorem 5.2). As a consequence we obtain the following result.

Corollary 1.2. (Corollary 5.5) The wild part of the inertia conjecture is true for an $n$-fold product $A_{d_{1}} \times \cdots \times A_{d_{n}}$ where $p \leq d_{i}<2$ for all $1 \leq i \leq n$.

In fact we show that if $\left(A_{r p},\langle\tau\rangle\right)$ and $\left(A_{r p+1},\langle\tau\rangle\right)$ are realizable for all $r \geq 2$ where $\tau$ is the product of $r$ disjoint $p$-cycles, then the wild part of the inertia conjecture holds for any finite product of Alternating groups.

The strategy of the proof is as follows. In [2, Section 21] Abhyankar introduced covers using some explicit equations and determined the Galois groups to be $A_{d}$, but the structure of the inertia group of such covers for $d \geq 2 p$ has not been studied. For Alternating groups $A_{d}$, we compute these inertia groups and use Abhyankar's Lemma to prove that $\left(A_{d}, I\right)$ is realizable if $I$ is generated by a $p$-cycle. For Theorem 5.2 we use Harbater's formal patching techniques to construct covers with the inertia group of the form $(\mathbb{Z} / p)^{2}$ with a desirable ramification filtration so that one could apply [13, Theorem 3.7] and reduce the inertia group.

In Section $\S 2$ we introduce basic definitions and results regarding inertia groups and the Galois covers of smooth projective curves. Section $\S 3$ contains some useful formal patching results needed to prove the main results. In Section $\$ 4$ we consider Alternating group covers. In Section $\S 5$ we treat the product of Alternating groups. Finally in Section $\$$ 6 for $d$, a multiple of $p$, we provide an explicit affine equation and show that its Galois closure is an $A_{d}$-Galois cover of $\mathbb{P}^{1}$ étale away from $\infty$. The inertia group and related invariants are also calculated for this cover. We also show that this $A_{d}$-cover has the minimum possible upper jump (in the sense of [5]).

Acknowledgements. To appear after the review process.

\section{Preliminaries}

Let $k$ be an algebraically closed field of characteristic $p>0$. A cover of $k$-curves means a finite generically separable morphism $\phi: Y \rightarrow X$ of smooth connected $k$-curves which is étale outside a finite set of closed points in $X$. The automorphism group $\operatorname{Aut}(Y / X)$ of a cover $\phi: Y \rightarrow X$ is the group of $k$-automorphisms $\sigma$ of $Y$ such that $\phi \circ \sigma=\phi$. For a finite group $G$, a $G$-Galois cover is a cover $Y \rightarrow X$ of curves together with an inclusion $\rho: G \hookrightarrow \operatorname{Aut}(Y / X)$ such that $G$ acts simply transitively on each generic geometric fibre. Since $X$ is irreducible, the inclusion $\rho$ is necessarily an isomorphism.

2.1. Group theory. The following group theoretic results are useful to decide which subgroups of $A_{d}$ or of a product of Alternating groups are potentially inertia subgroups. A 
special case $(r=1$ and $d<2 p)$ of the next result can be found in [14, Lemma 4.13, Lemma 4.14].

Proposition 2.1. Let $p$ be a prime, $d \geq p$. Let $\tau$ be an element of order $p$ in the Symmetric group $S_{d}$. Let $\tau=\prod_{i=1}^{r} \tau_{i}$ be the disjoint cycle decomposition of $\tau$ with $\tau_{1}, \cdots, \tau_{r}$ disjoint $p$-cycles and $r \cdot p \leq d$. For $\sigma \in S_{d}$ let $\operatorname{Supp}(\sigma)$ denote the support of $\sigma$. Then there exists an element $\theta \in \operatorname{Sym}(\operatorname{Supp}(\tau)) \cap N_{S_{d}}(\langle\tau\rangle)$ of order $p-1$ such that conjugation by $\theta$ is a generator of Aut $(\langle\tau\rangle)$. Moreover, let $H^{\prime}:=\left\{\sigma \in S_{d}: \operatorname{Supp}(\sigma) \subset \operatorname{Supp}(\tau), \sigma \cdot \tau_{i} \cdot \sigma^{-1}=\right.$ $\tau_{j}$ for $\left.1 \leq i, j \leq r\right\}$. Then $N_{S_{d}}(\langle\tau\rangle)=\left\langle\theta, H^{\prime}\right\rangle \times H$, where $H$ is the Symmetric group on the set $\{1, \cdots, d\} \backslash \operatorname{Supp}(\tau)$.

In particular, if $\beta \in N_{S_{d}}(\langle(1, \cdots, p)\rangle)$ has order prime to $p$ then $\beta=\theta^{i} \cdot \omega$ for some integer $0 \leq i \leq p-1$ and an element $\omega \in H=\operatorname{Sym}(\{p+1, \cdots, d\})$.

Proof. For a $p$-cycle $\tau^{\prime}$ in $S_{p}$, the normalizer $N_{S_{p}}\left(\left\langle\tau^{\prime}\right\rangle\right)$ is the affine general linear group $A G L(1, p)$ of order $p(p-1)$ and has an element of order $p-1$. Diagonally embedding $S_{p}$ in $\operatorname{Sym}(\operatorname{Supp}(\tau))$ we obtain an element $\theta$ of order $p-1$ in $\operatorname{Sym}(\operatorname{Supp}(\tau))$. Then $\theta$ normalizes $\langle\tau\rangle$ and the conjugation by $\theta$ has order $p-1$. Since $\tau$ is of order $p$, the full automorphism group of $\langle\tau\rangle$ is generated by $\theta$. So the natural homomorphism $N_{\operatorname{Sym}(\operatorname{Supp}(\tau))}(\langle\tau\rangle) \rightarrow \operatorname{Aut}(\langle\tau\rangle)$ is a surjection whose kernel is the centralizer of $\langle\tau\rangle$ in $\operatorname{Sym}(\operatorname{Supp}(\tau))$. Now observe that the centralizer of $\langle\tau\rangle$ in $\operatorname{Sym}(\operatorname{Supp}(\tau))$ is $H^{\prime}$. So $N_{S_{d}}(\langle\tau\rangle)=\left\langle\theta, H^{\prime}\right\rangle \times H$.

Notation 2.2. Let $u \geq 1$ and let $G=G_{1} \times \cdots \times G_{u}$ where each $G_{i}$ is a finite group. For $g \in G$, let $g=\left(g^{(i)}\right)_{1 \leq i \leq u}$, and set $S(g)=\left|\left\{1 \leq i \leq u \mid g^{(i)} \neq 1\right\}\right|$. Set $l(g)=|S(g)|$. For $\lambda \subseteq\{1, \cdots, u\}$, let $H_{\lambda}:=\prod_{i \in \lambda} G_{i}$ and let $\pi_{\lambda}: G \rightarrow H_{\lambda}$ be the projection.

Lemma 2.3. Let $u \geq 1$ be an integer, $G=G_{1} \times \cdots \times G_{u}$, where each $G_{i}$ is a simple non-abelian quasi p-group. Let $Q$ be a p-subgroup whose conjugates generate $G$. Then there are elements $g_{1}, \cdots, g_{r}$ in $Q$ for some $r \geq 1$ satisfying the following properties. Set $S_{\leq j}:=\cup_{i=1}^{j} S\left(g_{i}\right), 1 \leq j \leq r$.

(1) $S_{\leq r}=\{1, \cdots, u\}, l\left(g_{1}\right) \geq \cdots \geq l\left(g_{r}\right)$ and for all $1 \leq i, j \leq r, S\left(g_{i}\right) \cap S\left(g_{j}\right) \neq \varnothing$.

(2) For all $1 \leq i \leq r, H_{S_{\leq i}}$ is generated by the conjugates of $\left\langle g_{1}, \cdots, g_{i}\right\rangle$.

(3) For any subset $\left\{i_{1}, \cdots, i_{t}\right\} \subset\{1, \cdots, r\}$ and any integers $1 \leq a_{i_{1}}, \cdots, a_{i_{t}} \leq p-1$, $l\left(g_{i_{1}}^{a_{i_{1}}} \cdots g_{i_{t}}^{a_{i_{t}}}\right) \leq \max _{1 \leq j \leq t} l\left(g_{i_{j}}\right)$.

(4) For each $1 \leq i \leq r$, $\operatorname{ord}\left(g_{i}^{(j)}\right)=p$ for some $1 \leq j \leq u$.

Proof. Choose $g_{1} \in Q$ such that $l\left(g_{1}\right) \geq l(g)$ for all $g \in Q$. If $l\left(g_{1}\right)=u$ then set $r=1$ and note that conditions (1) and (3) trivially are satisfied. Condition (2) holds because $G_{j}$ 's are simple groups. Inductively define $g_{i}$ as follows. For $i \geq 1$, if $S_{\leq i}$ is not the whole set then choose $g_{i+1}$ among $g \in Q$ with $S(g) \cap\left(S_{\leq i}\right)^{c} \neq \varnothing$ such that $l\left(g_{i+1}\right)$ is maximal. The inclusion $S_{\leq i} \subset S_{\leq i+1}$ implies $l\left(g_{i}\right) \geq l\left(g_{i+1}\right)$. Since $G_{j}$ 's are all simple non-abelian groups, the conjugates of $\left\langle g_{1}, \cdots, g_{i}\right\rangle$ generate $H_{S_{\leq i}}$. For $j \leq i-1, l\left(g_{i} g_{j}\right) \leq l\left(g_{j}\right)$ and hence $S\left(g_{i}\right) \cap S\left(g_{j}\right) \neq \varnothing$. Condition (3) follows by the choice of $g_{j}$ 's to maximize $l\left(g_{j}\right)$ 's.

Finally for condition (4) note that if $p^{k+1}$ is the least order of $g_{i}^{(j)}$ for various $j$ among nontrivial $g_{i}^{(j)}$ then replacing $g_{i}$ by $g_{i}^{p^{k}}$ will achieve the goal.

Remark 2.4. In the above lemma if $1 \leq u \leq p$ then $r=1$, i.e. there exist $g \in Q$ such that $S(g)=\{1, \cdots, u\}$. Also note that we can take each $l\left(g_{i}\right) \geq p$.

The following lemma follows from the fact that the abelianization of a quasi $p$-group is a $p$-group.

Lemma 2.5. Let $G$ be a finite quasi p-group. Then the following are equivalent. 
(1) $G$ is perfect (i.e. $G$ equals its commutator subgroup).

(2) There is no nontrivial homomorphism from $G$ to $\mathbb{Z} / p$.

(3) There is no nontrivial homomorphism from $G$ to any p-group.

2.2. Ramification. Let $X$ be a smooth projective curve over $k$, with function field $K$. Let $F / K$ be a finite separable extension. Let $\psi: Y \rightarrow X$ be the normalization of $X$ in $F$. Let $\phi: \widetilde{Y} \rightarrow X$ be the Galois closure of $\psi$ with Galois group $G$. It is well-known ([14, Lemma 4.2]) that the branch loci of $\phi$ and $\psi$ are the same, which we denote by $B$. For details on the ramification theory for local extensions see [19, Chapter IV], from which we recall a few facts.

Let $x \in B, y \in \phi^{-1}(x)$, and let $I$ be the inertia group at $y$. Since $k$ is algebraically closed and $y$ is a closed point of $Y$, the inertia group is the same as the decomposition group at $y$. Note that $I$ is of the form $P \rtimes \mu_{m}$ where $P$ is a $p$-group and $(m, p)=1([19$. Chapter IV, Corollary 4]). Also recall that the lower ramification groups $\left\{I_{i}\right\}_{i \geq 0}$ constitute a finite decreasing filtration of $I$ at $y$ which is in an explicit bijection with the upper indexed ramification filtration of $I$. If $|I|=p m, p \nmid m$, there is a unique lower jump $h \geq 1$ which is called the conductor. The corresponding upper jump is denoted by $\sigma:=h / m$.

We fix the following setup for the rest of the section. Let $p>2$ be a prime, $d \geq p+2$ an integer and $\psi: Y \rightarrow \mathbb{P}^{1}$ a degree- $d$ smooth connected cover étale away from $\infty$. Let $\phi: \widetilde{Y} \rightarrow \mathbb{P}^{1}$ be its Galois closure with Galois group $G$. Note that $G$ is a transitive quasi $p$-subgroup of $S_{d}$. Assume that the inertia group at a point in $\widetilde{Y}$ lying over $\infty$ is $I=P \rtimes \mu_{m}$, where $P \cong \mathbb{Z} / p \mathbb{Z}$. Let $\tau$ be a (fixed) generator of $P$, and $\beta \in \mu_{m}$ is of order $m$. For the rest of this section we also assume that $\tau$ is the $p$-cycle $(1, \cdots, p)$. Let $h$ be the conductor. Also $I=N_{I}(\langle\tau\rangle) \subseteq N_{G}(\langle\tau\rangle)=N_{S_{d}}(\langle\tau\rangle) \cap G$. By Proposition 2.1 $\beta=\theta^{i} \omega$ for some $\theta$, $\omega \in N_{S_{d}}(\langle\tau\rangle)$ and $0 \leq i \leq p-1$. Furthermore $\theta \in \operatorname{Sym}(\operatorname{Supp}(\tau))=S_{p}$ is of order $p-1$ and $\omega \in \operatorname{Sym}\left(\operatorname{Supp}(\tau)^{c}\right)$.

Consider the group homomorphism $g:\langle\beta\rangle \rightarrow$ Aut $(\langle\tau\rangle), g(\beta): \tau \mapsto \beta \tau \beta^{-1}$. Let $m^{\prime}$ be the order of $\operatorname{ker}(g)=\left\{\beta^{j} \mid \beta^{j}\right.$ commutes with $\left.\tau\right\}$. This is the prime-to- $p$ part of the center of $G_{0}$, called the central part of the tame ramification. Put $m^{\prime \prime}:=\frac{m}{m^{\prime}}$. Then $\operatorname{ker}(g)=\left\langle\beta^{m^{\prime \prime}}\right\rangle$ is the subgroup acting trivially on $\langle\tau\rangle$. Also observe that $\operatorname{Im}(g)$ has order $m^{\prime \prime}$ in $\operatorname{Aut}(\langle\tau\rangle)$, and $m^{\prime \prime}$ is called the faithful part of the tame ramification. Let $h$ be the conductor. The following two lemmas are easy generalizations of results in [8, Section 4.6, Proposition 4.16 and 4.17].

Lemma 2.6. Under the above notation $m^{\prime}=$ g.c.d. $(h, m)$ and $\operatorname{ord}\left(\theta^{i}\right)=m^{\prime \prime}$.

Proof. Set $\gamma:=(h, m)$. Applying [19, Chapter IV, Proposition 9] to the conjugation of $\tau$ by $\beta^{\frac{m}{\gamma}}$ and $\beta^{m^{\prime \prime}}$, we get that $\beta^{\frac{m}{\gamma}} \tau \beta^{-\frac{m}{\gamma}}=\tau$ and $\beta^{m^{\prime \prime} h}=1$. So $m^{\prime \prime} \operatorname{divides} \frac{m}{\gamma}=\frac{m^{\prime} m^{\prime \prime}}{\gamma}$ and $m^{\prime} m^{\prime \prime}$ divides $m^{\prime \prime} h$. So $\gamma$ divides $m^{\prime}$ and $m^{\prime}$ divides $(h, m)=\gamma$. Thus $\gamma=m^{\prime}$.

Note that the conjugation by $\theta$ has order $p-1$ (Proposition 2.1). Since $\theta$ also has order $p-1, \theta^{j}$ commutes with $\tau$ if only if $\theta^{j}$ is the identity. Note that $\beta=\theta^{i} \cdot \omega$ and $\omega$ commutes with $\tau$. So the conjugation by $\beta$ is the same as the conjugation by $\theta^{i}$. Hence $\left(\theta^{i}\right)^{k}$ does not commute with $\tau$ for $0<k<m^{\prime \prime}$, and $\left(\theta^{i}\right)^{m^{\prime \prime}}$ commutes with $\tau$. So $m^{\prime \prime}$ is the order of $\theta^{i}$.

Since $\tau$ and $\theta$ are elements in $S_{p}$, the action of $I$ on $\{p+1, \cdots, d\}$ is the same as that of $\beta$ and hence is the same as the action of $\omega$. Suppose that the action of $\beta$ on $\{p+$ $1, \cdots, d$ \} breaks into $t$ disjoint cycles of lengths $n_{1}, \cdots, n_{t}$ (equivalently, the disjoint cycle decomposition of $\omega \in \operatorname{Sym}\{p+1, \cdots, d\}$ consists of $t$ disjoint cycles of length $\left.n_{1}, \cdots, n_{t}\right)$. The following result was proved in [8] with the assumption that the order of the group $G$ is strictly divisible by $p$. We note that the same proof works without this assumption on $|G|$ in view of Proposition 2.1 
Lemma 2.7. (cf. [8, Proposition 4.16]) Under the above notation the fibre of $\psi: Y \rightarrow \mathbb{P}^{1}$ above $\infty$ consists of $t+1$ points with ramification indices of order $p, n_{1}, \cdots, n_{t}$.

Since the action of $\beta$ on $\{p+1, \cdots, d\}$ is the same as that of $\omega \in H, \omega$ is a product of $t$ disjoint cycles of lengths $n_{1}, \cdots, n_{t}$. Set $L:=$ L.c.m. $\left(n_{1}, \cdots, n_{t}\right)$. Also since $\beta$ has order prime-to- $p$, for each $i,\left(p, n_{i}\right)=1$. Let $g(Y)$ be the genus of the curve $Y$. Using the Riemann-Hurwitz formula and Hilbert's different formula ([19, IV, Proposition 4]) to the Galois covers $\psi: Y \rightarrow \mathbb{P}^{1}$ and to $\widetilde{Y} \rightarrow Y$ (a technique used in [5, Proposition 1.3] when $p$ strictly divides the order of $G$ ), we obtain

$$
\sigma=\frac{h}{m}=\frac{2 g(Y)+d+t-1}{p-1} .
$$

By Lemma2.6, $m^{\prime \prime}$ is the smallest positive integer such that $m^{\prime \prime} \cdot \sigma$ is again an integer. So we have

$$
m^{\prime \prime}=\frac{p-1}{(p-1,2 g(Y)+d+t-1)} .
$$

Since order of $\theta^{i}$ is $m^{\prime \prime}$ by Lemma 2.6 and $m^{\prime} m^{\prime \prime}=m=1 . c . m$. $\left(\right.$ ord $\left(\theta^{i}\right)$, ord $\left.(\omega)\right)$, we deduce that

$$
m^{\prime}=\frac{L}{\left(L, m^{\prime \prime}\right)}
$$

Let us summarize the above observations in the following Theorem.

Theorem 2.8. Let $p$ be an odd prime, $d \geq p+2$, and $G$ be a transitive subgroup of $S_{d}$. Let $\tau \in G$ be a p-cycle, $P=\langle\tau\rangle$. Let $\phi: \widetilde{Y} \rightarrow \mathbb{P}^{1}$ be a $G$-Galois cover étale away from $\infty$, with inertia group $I=P \rtimes \mu_{m}$ above $\infty,(p, m)=1$. Let $Y$ denote the quotient $\widetilde{Y} /\left(G \cap S_{d-1}\right)$, and $\psi: Y \rightarrow \mathbb{P}^{1}$ be the degree-d cover. If $\beta$ is a generator of the cyclic group $\mu_{m}$, then $\beta$ is of the form $\theta^{i} \cdot \omega$, for some $1 \leq i \leq p-1$, where $\theta$ and $\omega$ are as in Proposition 2.1 There are prime-to-p integers $n_{1}, \cdots, n_{t}, t \geq 1(t \geq 2$ when $p \mid d)$, such that $\omega$ is a disjoint product of t-many cycles of length $n_{i}$ 's, and there are exactly $t+1$ points in the fibre of $\psi$ above $\infty$ with ramification indices $p, n_{1}, \cdots, n_{t}$. Moreover, the the upper jump $\sigma=h / m$, and the integers $m^{\prime \prime}, m^{\prime}$ are described by the Equations 2.1] 2.2 and 2.3. respectively.

\section{FORMAL PATCHING}

We fix the following notation.

Notation 3.1. Let $R=k[[t]], \widetilde{K}=k((t)), U=\operatorname{Spec}\left(k\left[\left[x^{-1}\right]\right]\right)$. Let $b$ be the closed point of $U$. For any $k$-algebra $A$ with $K=Q F(A)$ and any $k$-scheme $W$, let $W_{A}:=W \times_{k} A$, $W_{K}:=W_{A} \times_{A} K$, and for any closed point $w \in W, w_{A}:=w \times_{k} A, w_{K}:=w_{A} \times_{A} K$. For an $R$-scheme $V$, let $V^{0}$ denote the closed fiber of $V \rightarrow \operatorname{Spec}(R)$.

Given a Galois cover $f: Y \rightarrow X$ with inertia group $I$ at a point $y \in Y$ and $f(y)=x$, we deform the local $I$-Galois cover at $x$ to obtain a new cover with desired local properties. We use a formal patching argument (cf. [6, Theorem 2] or [7, Theorem 3.6]) to first get a cover over $X_{R}$ and then use a Lefschetz type principle to obtain Galois covers of smooth projective $k$-curves.

Lemma 3.2. (Formal Patching) Let $G$ be a finite group and $X$ be an irreducible smooth projective $k$-curve. Let $I_{1} \subset I$ and $H$ be subgroups of $G$ such that $G=\langle H, I\rangle$. Assume that there is an irreducible $H$-Galois cover $\phi: Y \rightarrow X$ of smooth projective curves branched only at a point $x \in X$, with inertia group $I_{1}$ at a point $y$ in $Y$ above $x$. Let $U_{X, x}=\operatorname{Spec}\left(\widehat{O}_{X, x}\right)$, 
$K_{X, x}=Q F\left(\widehat{O}_{X, x}\right)$ and $L=Q F\left(\widehat{O}_{Y, y}\right)$. Let $b_{x}$ denote the closed point of $U_{X, x}$. Let $Z \rightarrow U_{X, x, R}$ be an irreducible I-Galois cover of integral $R$-schemes totally ramified over $b_{x, R}$ such that the normalization of the pullback of $Z^{0} \rightarrow U_{X, x}$ to $\operatorname{Spec}\left(K_{X, x}\right)$ is isomorphic to $\operatorname{Ind}_{I_{1}}^{I} \operatorname{Spec}(L)$ as the I-Galois covers of $\operatorname{Spec}\left(K_{X, x}\right)$. Then there is a normal G-Galois cover $V \rightarrow X_{R}$ of irreducible R-curves such that the following hold.

(1) $V \rightarrow X_{R}$ is étale away from $x_{R}$ with inertia group I above $x_{R}$;

(2) $V \times_{X_{R}} U_{X, x, R} \cong \operatorname{Ind}_{I}^{G} Z$ and if $X^{\prime}=X \backslash\{x\}$ then $V \times_{X_{R}} X_{R}^{\prime} \cong \operatorname{Ind}_{H}^{G} Y_{R}^{\prime}$ where $Y^{\prime}=\phi^{-1}\left(X^{\prime}\right)$

(3) the closed fibre $V^{0}$ is connected and the normalization of the pullback of $V^{0} \rightarrow X$ to $\operatorname{Spec}\left(K_{X, x}\right)$ is isomorphic to $\operatorname{Ind}_{I_{1}}^{G} \operatorname{Spec}(L)$ as G-Galois cover of $\operatorname{Spec}\left(K_{X, x}\right)$.

Proof. Consider the trivial deformation $Y_{R}^{\prime} \rightarrow X_{R}^{\prime}$ of the $H$-Galois cover $Y^{\prime} \rightarrow X^{\prime}$. Taking disjoint union of $[G: H]$-copies of $Y_{R}^{\prime}$, we obtain a (disconnected) normal $G$-Galois cover $W_{1}:=\operatorname{Ind}_{H}^{G} Y_{R}^{\prime} \rightarrow X_{R}^{\prime}$, in which the stabilizers of the components are the conjugates of $H$ in $G$. Now taking disjoint union of $[G: I]$-copies of $Z$, we obtain a (disconnected) normal $G$-Galois branched cover $W_{2}:=\operatorname{Ind}_{I}^{G} Z \rightarrow U_{X, x, R}$, in which the stabilizers of the components are the conjugates of $I$ in $G$. The fibre of $W_{1}^{0}$ over $\operatorname{Spec}\left(K_{X, x}\right)$ is given by $\operatorname{Ind}_{H}^{G} \operatorname{Ind}_{I_{1}}^{H} \operatorname{Spec}(L)$ and that of $W_{2}^{0}$ over $\operatorname{Spec}\left(K_{X, x}\right)$ is given by $\operatorname{Ind}_{I}^{G} \operatorname{Ind}_{I_{1}}^{I} \operatorname{Spec}(L)$. Since both of these covers are indexed by left cosets of $I_{1}$ in $G$, we can choose an isomorphism between these fibres that is compatible with the indexing, and hence with the $G$-action. Since the pullbacks of $W_{1} \rightarrow X_{R}^{\prime}$ and $W_{2} \rightarrow U_{X, x, R}$ to $\operatorname{Spec}\left(K_{X, x, R}\right)$ are étale, by [18, I, Corollary 6.2], they are trivial deformations of the pullbacks of $W_{1}^{0} \rightarrow X^{\prime}$ and $W_{2}^{0} \rightarrow U_{X, x}$ to $\operatorname{Spec}\left(K_{X, x}\right)$. So the above isomorphism lifts uniquely to a $G$-isomorphism $W_{1} \times_{X_{R}^{\prime}} K_{X, x, R} \cong$ $W_{2} \times_{U_{X, x, R}} K_{X, x, R}$ over $\operatorname{Spec}\left(K_{X, x, R}\right)$. By [6, Proposition 4(b)], there exists a unique normal $G$-Galois cover $V \rightarrow X_{R}$ such that $V \times_{X_{R}} X_{R}^{\prime} \cong W_{1}$ and $V \times_{X_{R}} U_{X, x, R} \cong W_{2}$ as covers of $X_{R}^{\prime}$ and $U_{X, x, R}$ respectively. Since $W_{2}$ has branch locus $x_{R}$ and $W_{1}$ is étale, $V \rightarrow X_{R}$ has branch locus $\left\{x_{R}\right\}$. Also since $Z \rightarrow U_{X, x, R}$ is totally ramified above $b_{x, R}$, we have $\left.\left.\operatorname{Gal}\left(V \times_{X_{R}}\left(K_{X, x} \otimes_{k} \widetilde{K}\right)\right) /\left(K_{X, x} \otimes_{k} \widetilde{K}\right)\right)=\operatorname{Gal}\left(Z \times_{U_{X, x, R}}\left(K_{X, x} \otimes_{k} \widetilde{K}\right)\right) /\left(K_{X, x} \otimes_{k} \widetilde{K}\right)\right)=I$. So the inertia group above $x_{R}$ is $I$. Finally, since the the stabilizers of the identity components of $W_{1} \rightarrow X_{R}^{\prime}$ and of $W_{2} \rightarrow U_{X, x, R}$ are $H$ and $I$ respectively, the stabilizer of the identity component of $V \rightarrow X_{R}$ is $\langle H, I\rangle=G$. So $V$ is irreducible.

Lemma 3.3. Let $X$ be an irreducible smooth projective $k$-curve, and $x \in X$ be a closed point. Let $U_{X, x}=\operatorname{Spec}\left(\widehat{O}_{X, x}\right)$ and $K_{X, x}=Q F\left(\widehat{O}_{X, x}\right)$. Let $b_{x}$ denote the closed point of $U_{X, x}$. For $1 \leq i \leq r$, let $G_{i}$ be a finite group and let $I_{i}$ be a subgroup of $G_{i}$. Assume that for each $1 \leq i \leq r$, there is an $I_{i}$-Galois cover $\phi_{i}: \widetilde{S}_{i} \rightarrow U_{X, x, k[t]}$ of integral schemes with branch locus $b_{x, k[t]}$ over which it is totally ramified. For those closed points $\beta \in \mathbb{A}_{t}^{1}$ where the fiber $\widetilde{S}_{i, \beta}$ of $\widetilde{S}_{i} \rightarrow \mathbb{A}_{t}^{1}$ is integral, let $M_{\beta}^{(i)} / K_{X, x}$ denote the field extension corresponding to the cover $\widetilde{S}_{i, \beta} \rightarrow U_{X, x}$ at $t=\beta$. For $1 \leq i \leq r$, let $Z_{i}:=\widetilde{S}_{i} \times_{U_{X, x, k[l]}} U_{X, x, R}$, and $V_{i} \rightarrow X_{R}$ be a normal $G_{i}$-Galois cover of irreducible $R$-curves étale away from $x_{R}$ with inertia group $I_{i}$ above $x_{R}$ such that $V_{i} \times_{X_{R}} U_{X, x, R} \cong \operatorname{Ind}_{I_{i}}^{G_{i}} Z_{i}$. Then there is an open dense subset $\mathcal{V}$ of $\mathbb{A}_{t}^{1}$ such that for all closed points $(t=\beta)$ in $\mathcal{V}$ the following holds. For each $1 \leq i \leq r$, there is a $G_{i}$-Galois cover $W_{i} \rightarrow X$ branched only at $x$ with inertia group $I_{i}$ at a point in $W_{i}$ above $x$ such that the local $I_{i}$-Galois extension corresponding to the formal neighbourhood at that point is $M_{\beta}^{(i)} / K_{X, x}$.

Proof. Let $1 \leq i \leq r$. Consider the $G_{i}$-Galois covers $f^{(i)}: V_{i} \times_{X_{R}} U_{X, x, R} \rightarrow U_{X, x, R}$ and $g^{(i)}: \operatorname{Ind}_{I_{i}}^{G_{i}} Z_{i} \rightarrow U_{X, x, R}$. Since $f^{(i)}$ and $g^{(i)}$ are finite morphisms and are $G$-Galois covers, the isomorphism $V_{i} \times_{X_{R}} U_{X, x, R} \cong \operatorname{Ind}_{I_{i}}^{G_{i}} Z_{i}$ is equivalent to a $G_{i}$-equivariant isomorphism 
of coherent sheaves $f_{*}^{(i)}\left(O_{V_{i} \times X_{R}} U_{X, x, R}\right)$ and $g_{*}^{(i)}\left(O_{\operatorname{Ind}_{I_{i}}^{G_{i}} Z_{i}}\right)$ over $U_{X, x, R}$, and hence it is defined locally by matrices involving only finitely many functions over $U_{X, x, R}$. So there exists a finite type $k[t]$-algebra $A \subset R$ having smooth connected spectrum $E=\operatorname{Spec}(A)$ and for each $1 \leq i \leq r$, an irreducible $G_{i}$-Galois cover $\pi_{i}: F_{i} \rightarrow X_{A}$ branched only over $x_{E}$ with inertia group $I_{i}$ above $x_{E}$, together with an isomorphism $F_{i} \times_{X_{A}} U_{X, x, A} \cong \operatorname{Ind}_{I_{i}}^{G_{i}} \widetilde{S}_{i} \times_{U_{X, x, k[t]}} U_{X, x, A}$ such that $F_{i} \times_{A} R \cong V_{i}$, and the fibre over each closed point of $E$ is irreducible and non-empty. So for each point $e \in E$ and for each $1 \leq i \leq r, \widehat{F_{i, e}} \rightarrow X \times_{k}\{e\} \cong X$ is a $G_{i}$-Galois cover étale away from $x$ with inertia group $I_{i}$ above $x$, where $\widetilde{F_{i, e}}$ is the normalization of the fibre $F_{i, e}=\pi_{i}^{-1}\left(X \times_{k}\{e\}\right)$. Since the ring map $k[t] \rightarrow A$ is also injective, the finite type map $E \rightarrow \mathbb{A}_{t}^{1}$ is flat and dominant. So the image of $E$ in $\mathbb{A}_{t}^{1}$ is an open dense set which is our $\mathcal{V}$. Now for every point $\beta \in \mathcal{V}$ with preimage $e_{\beta} \in E$ and for each $1 \leq i \leq r$, the corresponding fibre $W_{i}:=\widetilde{F}_{i, e_{\beta}} \rightarrow X$ is a $G_{i}$-Galois cover branched only at $x$, and the $I_{i}$-Galois extension corresponding to the formal neighborhood of a point in $W_{i}$ lying above $x$ is $M_{\beta}^{(i)} / K_{X, x}$.

Remark 3.4. Note that the above two Lemmas 3.2, 3.3 can be easily generalized to the case with branch locus containing more than one point.

The following application of patching result by Raynaud ([17, Theorem 2.2.3]) will also be used later.

Theorem 3.5. (Raynaud) Let $G$ be a finite group and let $Q$ be a p-subgroup of $G$. Let $J$ be a finite set. For each $i \in J$, let $G_{i}$ be a subgroup of $G$ and $Q_{i}$ be a subgroup of $G_{i} \cap Q$. Assume that $G=\left\langle\left\{G_{i}\right\}_{i \in J}, Q\right\rangle$. Suppose that for each $i \in J$, there is a connected $G_{i}$-Galois étale cover of the affine line with inertia group $Q_{i}$ above $\infty$. Then there is a connected $G$-Galois étale cover of the affine line with inertia group $Q$ above $\infty$.

\section{Wild Part of the Inertia Conjecture for Alternating groups}

Let $p$ be an odd prime. In this section we prove the wild part of the inertia conjecture for Alternating group $A_{d}$ when the inertia group contains a $p$-cycle. Note that $A_{d}$ is a quasi $p$-group for $d \geq p$ and when $p \leq d<2 p$ the wild part of the conjecture is immediate. So we may assume that $d \geq 2 p$. We begin with the following lemma on the Galois groups of local fields. For a field $L$ and a separable polynomial $f(Z) \in L[Z]$, denote the Galois group of the splitting field of $f(Z)$ over $L$ by $\operatorname{Gal}(f, L)$.

Lemma 4.1. Let $h(Z) \in k((x))[Z]$ be an irreducible polynomial of degree $m$ where $(m, p)=$ 1. Then $\operatorname{Gal}(h, k((x)))=\mathbb{Z} / m \mathbb{Z}$.

Proof. Let $L=k((x))[Z] /(h(Z))$ and $L_{s}$ the splitting field of $h$ over $k((x))$. Let $G=$ $\operatorname{Gal}\left(L_{s} / k((x))\right)$ and $G^{\prime}=\operatorname{Gal}\left(L_{s} / L\right)$. Note that $G=P \rtimes \mu_{m}$ where $P$ is a $p$-group and $p \nmid m$. Since $[L: k((x))]=m, G^{\prime}$ is an index $m$ subgroup of $G$. But $(m, p)=1 \mathrm{im}-$ plies $P \leq G^{\prime}$. Hence $L \subset L_{s}^{P}$. But $L_{s}^{P} / k((x))$ is a Galois extension with cyclic Galois group. Hence $L / k((x))$ is also a cyclic Galois extension. So $L$ is the splitting field of $h$ and $\operatorname{Gal}(L / k((x)))=\mu_{m}$.

Definition 4.2. Let $G$ be a quasi $p$-group, and $I$ be a subgroup of $G$. Recall that the pair $(G, I)$ is realizable if there exists a $G$-Galois cover $Y \rightarrow \mathbb{P}^{1}$ branched only at $\infty$, with inertia group $I$ at a point above $\infty$. An $I$-Galois extension $L / k\left(\left(x^{-1}\right)\right)$ is said to be realized by the pair $(G, I)$ if there is a $G$-Galois cover of $\mathbb{P}^{1}$ branched only at $\infty$ with inertia group $I$ above $\infty$ so that the corresponding local $I$-Galois extension is isomorphic to $L / k\left(\left(x^{-1}\right)\right)$. 
By Harbater's result ([6, Theorem 2]), it is enough to show that $\left(A_{d},\langle\tau\rangle\right)$ is realizable when $\tau$ is a $p$-cycle. For this we use an equation of an $A_{d}$-cover given by Abhyankar.

Proposition 4.3. Let $p$ be an odd prime, $d=p+s \geq p+2, p \nmid s,(p, s) \neq(7,2)$. Consider the degree $d$ cover $\psi: Y \rightarrow \mathbb{P}^{1}$ given by the affine Equation

$$
x=\frac{1+y^{d}}{y^{s}} .
$$

Let $\phi: \widetilde{Y} \rightarrow \mathbb{P}^{1}$ be its Galois closure. Then $\phi$ is an $A_{d^{-}}$Galois cover of $\mathbb{P}^{1}$ branched only at $\infty$, with inertia group $I=\langle\tau\rangle \rtimes\langle\beta\rangle$ above $\infty$ where $\tau$ is a p-cycle, and $\beta$ has order l.c.m. $\left(\frac{p-1}{(p-1, s+1)}, s\right)$. Furthermore, it has upper jump $\frac{d}{p-1}$.

Proof. By [2, Section 11], $G=A_{d}$ and the cover $\phi$ is branched only at $\infty$. Set $x_{0}=x^{-1}$ and consider the local equation of $Y$ near a preimage of $x=\infty$. Then $I$ is the Galois group of the splitting field of $f(y)=x_{0} \cdot y^{d}-y^{s}+x_{0}$ over $k\left(\left(x_{0}\right)\right)$. By Hensel's Lemma, $f(y)=g(y) \cdot h(y)$ in $k\left[\left[x_{0}\right]\right][y]$ with $g(y) \equiv-1\left(\bmod x_{0}\right)$ of degree $p$ and $h(y) \equiv y^{s}\left(\bmod x_{0}\right)$ of degree $s$. So we have

$$
\mathrm{Gal}\left(f(y), k\left(\left(x_{0}\right)\right)\right) \leq \mathrm{Gal}\left(g(y), k\left(\left(x_{0}\right)\right)\right) \times \mathrm{Gal}\left(h(y), k\left(\left(x_{0}\right)\right)\right) .
$$

Let $h(y)=b_{0}+\cdots+b_{s} y^{s}$ for some $b_{i} \in k\left[\left[x_{0}\right]\right]$. Then $\operatorname{ord}\left(b_{i}\right) \geq 1$ for $1 \leq i \leq s-1$, and $\operatorname{ord}\left(b_{s}\right)=0$. Since $f=g \cdot h, b_{0}$ divides $x_{0}$, and so $\operatorname{ord}\left(b_{0}\right)=1$. So $h(y)$ is Eisenstein, and hence it is an irreducible polynomial in $k\left(\left(x_{0}\right)\right)$ [y]. Since $h$ is irreducible of prime-to- $p$ degree $s$, by Lemma 4.1. $\operatorname{Gal}\left(h, k\left(\left(x_{0}\right)\right)\right)=\mu_{s}$. Also $\operatorname{Gal}\left(g, k\left(\left(x_{0}\right)\right)\right) \leq \operatorname{Gal}\left(f, k\left(\left(x_{0}\right)\right)\right) \leq$ $\operatorname{Gal}\left(f, k\left(x_{0}\right)\right)=A_{d}$. Moreover, $p$ divides the order of the inertia group $I$ and $g$ has degree $p$ and so $\operatorname{Gal}\left(g, k\left(\left(x_{0}\right)\right)\right)$ is a transitive subgroup of $S_{p}$. Hence $\operatorname{Gal}\left(g, k\left(\left(x_{0}\right)\right)\right) \leq A_{p}$. So the Sylow $p$-subgroup $P$ of $\operatorname{Gal}\left(g, k\left(\left(x_{0}\right)\right)\right)$ is generated by a $p$-cycle $\tau$. Since $I \subset N_{A_{d}}(\langle\tau\rangle)=$ $N_{S_{d}}(\langle\tau\rangle) \cap A_{d}$, by Proposition 2.1, the inertia group $I$ is of the form $\langle\tau\rangle \rtimes\langle\beta\rangle$, with $\beta=\theta^{i} \cdot \omega$ for an element $\theta$ of order $(p-1)$, an integer $i$ and $\omega \in H=\operatorname{Sym}(\{p+1, \cdots, d\})$. Since $x$ is a rational function of $y, Y \cong \mathbb{P}^{1}$. Note that there are exactly two points in the fibre $\psi^{-1}(\infty)$ with ramification indices $p$ and $s$. So by Lemma 2.7, $\omega$ is an $s$-cycle. Let $h$ be the conductor and let $\beta$ has order $m$. Then by Equation 2.1 the upper jump $\frac{h}{m}=\frac{d}{p-1}$. Now Equation 2.2 implies $\operatorname{ord}\left(\theta^{i}\right)=m^{\prime \prime}=\frac{p-1}{(p-1, d)}=\frac{p-1}{(p-1, s+1)}$. So we have $m=1$.c.m. $\left(\operatorname{ord}\left(\theta^{i}\right), \operatorname{ord}(\omega)\right)=$ 1.c.m. $\left(\frac{p-1}{(p-1, s+1)}, s\right)$.

Observe that the above Proposition 4.3 was proved in [14, Theorem 4.9] using a different method when $s<p$.

Corollary 4.4. Let $d=p+s, p \nmid s$. Then for any $p$-cycle $\tau$, the pair $\left(A_{d},\langle\tau\rangle\right)$ is realizable.

Proof. By Proposition 4.3, the $A_{d}$-Galois cover of $\mathbb{P}^{1}$ obtained from Equation (4.1) is branched only at $\infty$ with inertia group $I$ whose Sylow $p$-subgroup is generated by a $p$ cycle. Now the result follows by applying Abhyankar's Lemma to this cover.

Corollary 4.5. Let $d=$ ap for some integer $a \geq 1$. Then for any $p$-cycle $\tau$, the pair $\left(A_{d},\langle\tau\rangle\right)$ is realizable.

Proof. We may assume that $a \geq 2$. Consider the $A_{d+1}$-Galois cover $\phi: \widetilde{Y} \rightarrow \mathbb{P}^{1}$ branched only at $\infty$, which is the Galois closure of the degree- $d$ cover $\psi: Y \rightarrow \mathbb{P}^{1}$ given by the affine Equation

$$
x=\frac{1+y^{d+1}}{y^{d-p+1}}
$$


By Proposition 4.3, the inertia group $I$ above $\infty$ is given by $\langle\tau\rangle \rtimes\langle\beta\rangle \cong \mathbb{Z} / p \rtimes \mu_{m}$ for a $p$-cycle $\tau$ and for the prime-to- $p$ integer $m=1$.c.m. $\left(\frac{p-1}{(p-1, d+1)}, d-p+1\right)$. Consider the $A_{d}$-Galois cover $\epsilon: \widetilde{Y} \rightarrow Y \cong \mathbb{P}^{1}$. By Lemma 2.7, this cover is branched at two points $(y=0)$ and $(y=\infty)$ with respective inertia groups of order $p \frac{m}{d-p+1}$ and $m$ above them. Let $\eta_{m}: \mathbb{P}^{1} \rightarrow \mathbb{P}^{1}$ be the $m$-cyclic Kummer cover by sending $z^{m}$ to $y$. Since $A_{d}$ is a quasi $p$-group, $\epsilon$ is an $A_{d}$-cover and $\eta_{m}$ is prime-to- $p$ Galois cover, they are linearly disjoint. By the refined Abhyankar's lemma ([14, Lemma 4.1]), the normalized pullback $Z \rightarrow \mathbb{P}^{1}$ of $\epsilon$ along $\eta_{m}$ is an $A_{d}$-Galois cover branched only at $\infty$, and the inertia group above $\infty$ is generated by the $p$-cycle $\tau$.

Remark 4.6. From Proposition 4.3, it follows that the conductor $h$ for the cover $\phi$ is given by $h=\frac{d+1}{p-1} \times 1$.c.m. $\left(\frac{p-1}{(p-1, d+1)}, d-p+1\right)$. By [19, IV, Proposition 2], it follows that the cover $\epsilon$ also has conductor $h$. Applying [16, Lemma 4.1] shows that the $A_{d}$-Galois cover $Z \rightarrow \mathbb{P}^{1}$ also has the same conductor $h$. In Section 6 we introduce a different cover which has the minimal possible upper jump $\frac{d+1}{p-1}$ and offers an alternate proof (cf. Proposition 6.3) for Corollary 4.5 when $a \geq 3$.

The following result follows immediately from Corollary 4.4 and Corollary 4.5 together with Harbater's result [6, Theorem 2].

Corollary 4.7. Let $P$ be a p-subgroup of $A_{d}$ containing a p-cycle. Then the pair $\left(A_{d}, P\right)$ is realizable.

Remark 4.8. Note that to prove the wild part of the inertia conjecture for $A_{d}$ one needs to show that $\left(A_{d},\langle\tau\rangle\right)$ is realizable where $\tau$ is a product of $r$-many disjoint $p$-cycles for all $r$ with $1 \leq r \leq\left\lfloor\frac{d}{p}\right\rfloor$.

The following result shows that to prove the wild part of the inertia conjecture for $A_{d}$, $d \geq p$, it is enough to prove the conjecture for the cases $d \equiv 0$ or $1(\bmod p)$.

Proposition 4.9. Let $r \geq 2$ be an integer. Assume that the pair $\left(A_{r p+1},\langle\tau\rangle\right)$ is realizable where $\tau$ is the product of $r$ disjoint $p$-cycles in $A_{r p+1}$. Then for any $d \geq r p+1$, the pair $\left(A_{d},\langle\tau\rangle\right)$ is realizable.

Proof. This is an immediate consequence of Raynaud's result (Theorem 3.5) by taking $G_{i}=\operatorname{Alt}(\operatorname{Supp}(\tau) \cup\{i\})$ and $Q=Q_{i}=\langle\tau\rangle$ for $i \in\{1, \cdots, d\} \backslash \operatorname{Supp}(\tau)$.

\section{Wild Part of the Inertia Conjecture for Product of Alternating groups}

The first two results of this section use formal patching results from section $\S 3$ to construct $G_{1} \times G_{2}$ covers of $\mathbb{P}^{1}$ from the given $G_{1}$ and $G_{2}$ covers such that the inertia group over $\infty$ is smaller than the one obtained from the fiber product of the two covers. This will be used to construct a product of Alternating group covers with a certain cyclic $p$-group as the inertia group. In view of Lemma 2.3 and [6, Theorem 2], this is exactly what we need.

Lemma 5.1. Let $G_{1}, G_{2}$ be two quasi p-groups, $P_{1}$ and $P_{2}$ be p-subgroups of $G_{1}$ and $G_{2}$ respectively. Assume that the pairs $\left(G_{1}, P_{1}\right)$ and $\left(G_{2}, P_{2}\right)$ are realizable, and let $Q_{1}$ and $Q_{2}$ be index-p subgroups of $P_{1}$ and $P_{2}$ respectively. Assume that the local $P_{1} / Q_{1}$ and $P_{2} / Q_{2}$ Galois extensions are given by the Artin-Schreier polynomials $f_{0}=Z_{1}^{p}-Z_{1}-f\left(x_{0}\right)$ and $g_{0}=Z_{2}^{p}-Z_{2}-g\left(x_{0}\right)$ respectively, where $x_{0}$ is the local parameter of $\mathbb{P}^{1}$ at $\infty$. Assume that $\operatorname{ord}_{x_{0}}(g)$ and $\operatorname{ord}_{x_{0}}(f)$ are different and not multiples of $p$. For $\alpha \in k$, let $M_{\alpha} / K$ and $N_{\alpha} / K$ be the $\mathbb{Z} / p$-Galois extensions given by the polynomials $f_{\alpha}=Z^{p}-Z-(1-$ $\alpha) f\left(x_{0}\right)-\alpha g\left(x_{0}\right)-\alpha x_{0}^{-1} \in k\left(\left(x_{0}\right)\right)[Z]$ and $g_{\alpha}=Z^{p}-Z-(1+\alpha) g\left(x_{0}\right)+\alpha f\left(x_{0}\right) \in k\left(\left(x_{0}\right)\right)[Z]$, 
respectively. Then there is a dense open subset $\mathcal{V}$ of $\mathbb{A}_{t}^{1}$ such that for all closed points $(t=\alpha)$ in $\mathcal{V}$, there exist a $P_{1}$-Galois extension $\widetilde{M}_{\alpha} / K$ and a $P_{2}$-Galois extension $\widetilde{N}_{\alpha} / K$ such that $M_{\alpha}=\widetilde{M}_{\alpha}^{Q_{1}}, N_{\alpha}=\widetilde{M}_{\alpha}^{Q_{2}}, \widetilde{M}_{\alpha} / K$ is realized by the pair $\left(G_{1}, P_{1}\right)$, and $\widetilde{N}_{\alpha} / K$ is realized by the pair $\left(G_{2}, P_{2}\right)$.

Proof. Let $U=\operatorname{Spec}\left(k\left[\left[x_{0}\right]\right]\right), R=k[[t]]$. Let $A:=k\left(\left(x_{0}\right)\right)[t][Z] /\left(f_{t}\right)$, where $f_{t}(Z)=$ $Z^{p}-Z-(1-t) f\left(x_{0}\right)-\operatorname{tg}\left(x_{0}\right)-t x_{0}^{-1} \in k\left(\left(x_{0}\right)\right)[t][Z]$ and $B:=k\left(\left(x_{0}\right)\right)[t][Z] /\left(g_{t}\right)$, where $g_{t}(Z)=Z^{p}-Z-(1+t) g\left(x_{0}\right)+t f\left(x_{0}\right) \in k\left(\left(x_{0}\right)\right)[t][Z]$. We have the maps $\phi_{\mathbb{A}_{t}^{\prime}}^{\prime}: S^{\prime}:=$ $\operatorname{Spec}(A) \rightarrow \operatorname{Spec}\left(k\left(\left(x_{0}\right)\right)[t]\right)$ and $\psi_{\mathbb{A}_{t}^{\prime}}^{\prime}: T^{\prime}:=\operatorname{Spec}(B) \rightarrow \operatorname{Spec}\left(k\left(\left(x_{0}\right)\right)[t]\right)$. Let $\phi_{\mathbb{A}_{t}^{1}}: S \rightarrow$ $U \times_{k} \mathbb{A}_{t}^{1}$ and $\psi_{\mathbb{A}_{t}^{1}}: T \rightarrow U \times_{k} \mathbb{A}_{t}^{1}$ be the normalization maps in $A$ and $B$ respectively. Let $\phi_{R}: S_{R} \rightarrow U_{R}$ and $\psi_{R}: T_{R} \rightarrow U_{R}$ be their pullbacks under the map $U_{R} \rightarrow U \times_{k} \mathbb{A}_{t}^{1}$. Then the normalization of the closed fibre of $\phi_{R}$ and $\psi_{R}$ correspond to the field extensions $M_{0} / k\left(\left(x_{0}\right)\right)$ and $N_{0} / k\left(\left(x_{0}\right)\right)$ respectively. The covers $\phi_{R}$ and $\psi_{R}$ are branched only at the $R$-valued point $x_{0}=0$ since it is the only pole of the functions $f_{t}$ and $g_{t}$ in $R\left[\left[x_{0}\right]\right]$. By [9, Theorem 3.11], there exist connected $P_{1}$ and $P_{2}$-Galois étale covers $\Phi_{\mathbb{A}_{t}^{\prime}}^{\prime}: \widetilde{S}^{\prime} \rightarrow \operatorname{Spec}\left(k\left(\left(x_{0}\right)\right)[t]\right)$ and $\Psi_{\mathbb{A}_{t}^{\prime}}^{\prime}: \widetilde{T}^{\prime} \rightarrow \operatorname{Spec}\left(k\left(\left(x_{0}\right)\right)[t]\right)$ dominating $\phi_{\mathbb{A}_{t}^{\prime}}^{\prime}$ and $\psi_{\mathbb{A}_{t}^{\prime}}^{\prime}$ respectively. Taking normalization of $U \times_{k} \mathbb{A}_{t}^{1}$ in the function fields of $\widetilde{S}^{\prime}$ and $\widetilde{T}^{\prime}$, we obtain $P_{1}$ and $P_{2}$-Galois covers $\Phi_{\mathbb{A}_{t}^{1}}: \widetilde{S} \rightarrow$ $U \times_{k} \mathbb{A}_{t}^{1}$ and $\Psi_{\mathbb{A}_{t}^{1}}: \widetilde{T} \rightarrow U \times_{k} \mathbb{A}_{t}^{1}$ dominating $\phi_{\mathbb{A}_{t}^{1}}$ and $\psi_{\mathbb{A}_{t}^{1}}$ respectively. One also obtains $\Phi_{R}$ and $\Psi_{R}$ which dominate $\phi_{R}$ and $\psi_{R}$ by pull backs.

So by Lemma 3.2, there are $G_{1}$ and $G_{2}$-Galois covers of $\mathbb{P}_{R}^{1}$ satisfying the hypothesis of Lemma3.3. So there is a dense open subset $\mathcal{V}$ of $\mathbb{A}_{t}^{1}$ such that for all points $(t=\alpha)$ in $\mathcal{V}$, the extension $\widetilde{M}_{\alpha} / K$ is realized by the pair $\left(G_{1}, P_{1}\right)$, and the extension $\widetilde{N}_{\alpha} / K$ is realized by the pair $\left(G_{2}, P_{2}\right)$.

Theorem 5.2. Let $G_{1}, G_{2}$ be two perfect quasi p-groups. Let $\tau \in G_{1}$ and $\sigma \in G_{2}$ be of order $p$ and $p^{r}$ for some $r$ respectively. Let $P_{1}=\langle\tau\rangle \leq G_{1}, P_{2}=\langle\sigma\rangle \leq G_{2}$. Assume that the pairs $\left(G_{1}, P_{1}\right)$ and $\left(G_{2}, P_{2}\right)$ are realizable. Then there exists $1 \leq a \leq p-1$ such that for $I:=\left\langle\left(\tau^{a}, \sigma\right)\right\rangle \leq G_{1} \times G_{2}$, the pair $\left(G_{1} \times G_{2}, I\right)$ is also realizable.

Proof. Let $\phi_{i}: Y_{i} \rightarrow \mathbb{P}^{1}$ be a $G_{i}$-Galois cover of $\mathbb{P}^{1}$ branched only at $\infty$ with inertia group $P_{i}$ above $\infty$ and conductor $h_{i}$, for $i=1,2$. As usual it can be arranged that $h_{2}<h_{1}$ and the first upper jump of the $P_{2}$-extension is at least 2 ([16, Theorem 2.2.2]. Let $N_{2}$ be the index $p$ subgroup of $P_{2}$. Let the local $P_{1}$ and $P_{2} / N_{2}$ Galois extensions be given by the Artin-Schreier polynomials $f_{0}=Z_{1}^{p}-Z_{1}-f\left(x_{0}\right) \in k\left(\left(x_{0}\right)\right)\left[Z_{1}\right]$ and $g_{0}=Z_{2}^{p}-Z_{2}-g\left(x_{0}\right) \in$ $k\left(\left(x_{0}\right)\right)\left[Z_{2}\right]$ respectively where $x_{0}$ is a local parameter at $\infty$. By Lemma 5.1] there is a dense open subset $\mathcal{V}$ of $\mathbb{A}_{t}^{1}$ such that for all points $(t=\alpha)$ in $\mathcal{V}$, the extension $M_{\alpha} / K$ given by the polynomial $f_{\alpha}=Z^{p}-Z-(1-\alpha) f\left(x_{0}\right)-\alpha g\left(x_{0}\right)-\alpha \cdot x_{0}^{-1}$ is realized by the pair $\left(G_{1}, P_{1}\right)$, and the extension $N_{\alpha} / K$ given by the polynomial $g_{\alpha}=Z^{p}-Z-(1+\alpha) g\left(x_{0}\right)+\alpha f\left(x_{0}\right)$ is dominated by a $P_{2}$-Galois extension $\widetilde{N}_{\alpha} / K$ which is realized by the pair $\left(G_{2}, P_{2}\right)$. So there is an $\alpha \neq 0,1$ such that the points $(t=\alpha)$ and $(t=\alpha-1)$ both lie in $\mathcal{V}$. Let $X_{i} \rightarrow \mathbb{P}^{1}$ be the corresponding $G_{i}$-Galois covers of the affine line with inertia groups $P_{i}$ above $\infty$, and $\eta_{i}$ be points in $X_{i}$ over $\infty$ such that $K_{X_{1}, \eta_{1}}=M_{\alpha}$ and $K_{X_{2}, \eta_{2}}=\widetilde{N}_{\alpha-1}$.

Let $a_{1} \in M_{\alpha}$ be a root of $f_{\alpha}$ and $a_{2} \in N_{\alpha-1}$ be a root of $g_{\alpha-1}$. So $a_{1}-a_{2}$ is a root of the Artin-Schreier equation $Z^{p}-Z+\alpha x_{0}^{-1}=0$. Since $-v_{x_{0}}\left(\left((1-\alpha) f\left(x_{0}\right)+\alpha g\left(x_{0}\right)+\right.\right.$ $\left.\left.\alpha x_{0}^{-1}\right)-\left(\alpha g\left(x_{0}\right)-(\alpha-1) f\left(x_{0}\right)\right)\right)=1$, by Theorem [12, Proposition 3.1], the compositum $M=M_{\alpha} \widetilde{N}_{\alpha}$ is a $Q=\mathbb{Z} / p \times \mathbb{Z} / p^{r}$-Galois extension with the first lower jump at 1 , and $Q_{1}=Q, Q_{2}=\operatorname{Gal}\left(M / K\left(a_{1}-a_{2}\right)\right) \cong \mathbb{Z} / p^{r}$. 
Let $X$ be the dominant connected component of the normalization of $X_{1} \times_{\mathbb{P} 1} X_{2}$ containing the point $\eta:=\left(\eta_{1}, \eta_{2}\right)$. Then $\Theta: X \rightarrow \mathbb{P}^{1}$ is a $G_{1} \times G_{2}$-Galois cover branched only above $\infty$ with the local extension $K_{X, \eta} / K_{\mathbb{P}^{1}, \infty}$ is given by $M / k\left(\left(x_{0}\right)\right)$, and the inertia subgroup $Q$ has a lower jump at 1 . Since $G_{1}$ and $G_{2}$ are perfect, $G_{1} \times G_{2}$ is also perfect, and so by [13, Theorem 3.7], there is a $G_{1} \times G_{2}$-Galois cover of the affine line with inertia group above $\infty$ given by $I=\operatorname{Gal}\left(M / K\left(a_{1}-a_{2}\right)\right)$ in $G$. Now the projection maps from $G_{1} \times G_{2}$ restricted to $I$ surjects onto $\operatorname{Gal}\left(M_{\alpha} / K\right)=P_{1}=\langle\tau\rangle$ and $\operatorname{Gal}\left(\widetilde{N}_{\alpha-1} / K\right)=P_{2}=\langle\sigma\rangle$. So $I$ is of the form $\left\langle\left(\tau^{a}, \sigma\right)\right\rangle$ for some $1 \leq a \leq p-1$.

Remark 5.3. Observe that a finite product of groups preserves the properties of being quasi $p$ and perfect. In particular, if $G=A_{d_{i}}, d_{i} \geq p, i=1,2$, then the hypothesis of the Theorem 5.2 is satisfied.

Corollary 5.4. Let $r \geq 2$ and $u \geq 1$ be integers. For $1 \leq i \leq u$, let $d_{i} \geq p$. Assume that the pairs $\left(A_{r p},\langle\tau\rangle\right)$ and $\left(A_{r p+1},\langle\tau\rangle\right)$ are realizable where $\tau$ is the product of $r$ disjoint $p$-cycles in $A_{r p}$. Then the wild part of the inertia conjecture is true for $A_{d_{1}} \times \cdots \times A_{d_{u}}$.

Proof. In view of Corollary 4.7 Proposition 4.9 and Remark 4.8 , the hypothesis implies that the wild part of inertia conjecture is true for Alternating groups. Set $G:=A_{d_{1}} \times \cdots \times A_{d_{u}}$. Let $P$ be a $p$-subgroup of $G$ whose conjugates generate $G$. There exist $g_{1}, \cdots, g_{r} \in P$ satisfying conditions (1)-(4) of Lemma 2.3.

The case $r=1$ will be proved by induction on $u$. Let $\pi_{i}: G \rightarrow A_{d_{i}}$ and $\pi: G \rightarrow$ $A_{d_{2}} \times \cdots \times A_{d_{u}}$ be the projection maps. So there exists a cyclic subgroup $P^{\prime}=\left\langle g_{1}\right\rangle$ of $P$ such that $\pi_{1}\left(P^{\prime}\right)=\langle\tau\rangle \leq A_{d_{1}}$ is a cyclic group of order $p, \pi\left(P^{\prime}\right)$ is a cyclic $p$-group with generator $\sigma$, say, and $\pi_{i}\left(P^{\prime}\right)$ are nontrivial subgroups for all $i$. By induction hypothesis on $u,\left(A_{d_{2}} \times \cdots A_{d_{u}}, \pi\left(P^{\prime}\right)\right)$ is realizable. Moreover $P^{\prime}=\langle(\tau, \sigma)\rangle$ and $\tau$ is of order $p$. Now by Theorem 5.2, $\left(A_{d_{1}} \times \cdots \times A_{d_{u}}, I\right)$ is realizable where $I=\left\langle\left(\tau^{a}, \sigma\right)\right\rangle$ for some $1 \leq a \leq p-1$. But there is an automorphism of $A_{d_{1}}$ which sends $\tau$ to $\tau^{a}$. Hence $\left(A_{d_{1}} \times \cdots \times A_{d_{u}}, P^{\prime}\right)$ is realizable. Finally by Harbater's result [6, Theorem 2], $\left(A_{d_{1}} \times \cdots \times A_{d_{u}}, P\right)$ is realizable.

Now for $r \geq 2$, in the Notation 2.2 of Lemma 2.3, let $H_{i}=H_{S\left(g_{i}\right)}$. By $r=1$ case, the pairs $\left(H_{i},\left\langle g_{i}\right\rangle\right)$ are realizable for $1 \leq i \leq r$. Now the result follows from Theorem 3.5 .

Corollary 5.5. Let $u \geq 1$ be an integer and let $p \leq d_{i}<2 p$ for all $1 \leq i \leq u$. The wild part of the inertia conjecture is true for $A_{d_{1}} \times \cdots \times A_{d_{u}}$. Moreover, if $Q$ is any $p$-group, the wild part of the inertia conjecture is true for $A_{d_{1}} \times \cdots \times A_{d_{u}} \times Q$.

Proof. When $d \leq 2 p-1$, any $p$-subgroup of $A_{d}$ contains a $p$-cycle. So the first part of the statement follows from Corollary 4.7 and Corollary 5.4. The second statement follows from the first one and [12, Corollary 4.6].

\section{A New Equation for Alternating Group covers}

Let $p$ be an odd prime and let $a \geq 3$ be an integer. Set $d=a p$. In this section we produce an $A_{d}$-Galois étale cover of the affine line with wild part of the inertia group generated by a $p$-cycle which occurs as the Galois closure of a degree- $d$ cover given by an explicit affine equation. Since there are ways to increase the upper jump of the ramification filtration, an interesting problem is to find covers with minimal possible upper jump. Riemann-Hurwitz formula provides a lower bound for the upper jump. We show that the resulting cover attains this lower bound.

Lemma 6.1. Let $p>2$ be a prime number, $d=a p, a \geq 3, j:=\frac{p-1}{(p-1, d+1)}$. Then there exists an integer $s, 1 \leq s \leq p-1$, such that $s \mid j$ and $(j, d-p-s)=1$. 
Proof. Since $j \mid(p-1)$, for any integer $s$ we have $(j, d-p-s)=(j, a-1-s)$. When $a=3$, for any $p>2, s=1$ satisfies the stated properties. For some $a$ and $p$, if $j=1$, then $s=1$ is again a solution. Now assume that $s$ satisfies the stated properties for some $p$ and $a$. Consider $a^{\prime}=a+p-1, d^{\prime}=a^{\prime} p$, and $j^{\prime}=\frac{p-1}{(p-1,(a+p-1) p+1)}$. Then $j^{\prime}=\frac{p-1}{(p-1, a+1)}=j$ and

$$
\left(j^{\prime}, d^{\prime}-p-s\right)=(j,(a+p-2) p-s)=(j,(a-1) p-s)=(j, d-p-s) .
$$

Since $(j, d-p-s)=1$ by assumption, $s$ is also a solution for $p$ and $a^{\prime}$.

So for a fixed $p>2$, we may assume that $4 \leq a \leq p+1$. Let $Q_{1}$ and $Q_{2}$ be the set of primes dividing $a+1$ and $a-1$ respectively. Let $Q$ be the set of primes dividing $p-1$, and for any prime $q \in Q$, let $\lambda_{q}$ be the highest positive integer such that $q^{\lambda_{q}} \mid(p-1)$. Let $I_{1}$ be the set of primes dividing $(p-1, a+1)$ and $I_{2}$ be the set of primes dividing $(p-1, a-1)$. Set $I:=I_{1} \cup I_{2}$. Let $(p-1, a+1)=\Pi_{q \in I_{1}} q^{m_{q}}$ and $a-1=\Pi_{q \in Q_{2}} q^{s_{q}}$ (we use the notion that an empty product equals 1). For $q \in I_{1} \backslash I_{2}$, set $\alpha_{q}:=m_{q}$ and for $q \in I_{2}$, set $\alpha_{q}:=\lambda_{q}$. So we have

$$
j=\frac{p-1}{(p-1, d+1)}=\frac{p-1}{(p-1, a+1)}=\frac{p-1}{\prod_{q \in I_{1}} q^{m_{q}}} .
$$

Let $s=\frac{p-1}{\Pi_{q \in I} q^{\alpha q}}$ and $v=\frac{\Pi_{q \in I} q^{\alpha q}}{\Pi_{q \in I_{1}} q^{m q}}$. Note that $s v=j$. Let

$$
g=(j, d-p-s)=(j, a-1-s)=\left(\frac{p-1}{\prod_{q \in I_{1}} q^{m_{q}}}, \Pi_{q \in Q_{2}} q^{s_{q}}-\frac{p-1}{\Pi_{q \in I} q^{\alpha_{q}}}\right) .
$$

Let $q$ be a prime dividing $g$. So $q \mid j=s v$. If $q \mid s$, then $q \notin I_{2}$ as $\alpha_{q}=\lambda_{q}$ for $q \in I_{2}$. Since $q$ divides both $j$ and $s$, it must divide $a-1$, and so $q \in I_{2}$, a contradiction. So $q \nmid s$ and we have $q \mid v$. So $q \in I_{2}$, and thus $q \mid(a-1)$. Thus $q \nmid(a-1-s)$ contradicting $q$ divides $g$. So we have $g=1$.

Remark 6.2. Let $p, a$ and $s$ be as in the above Lemma6.1. When $a=3$, we have $s=1$, and so $d-p-s=2 p-1>\frac{3 p}{2}$. For $a \geq 4$, we have $s \leq p-1$, and so $d-p-s \geq d-p-(p-1)>\frac{d}{2}$.

Now set $j:=\frac{p-1}{(p-1, d+1)}$. Using Lemma6.1, fix an integer $1 \leq s \leq p-1$, such that $s \mid j$ and $(j, d-p-s)=1$. Consider the degree- $d$ cover $\psi: Y \rightarrow \mathbb{P}^{1}$ given by the affine equation

$$
x=\frac{1+y^{d-s}(y+1)^{s}}{y^{d-p-s}(y+1)^{s}} .
$$

Put $h(y):=1+y^{d-s}(y+1)^{s} \in k[y]$. Writing the above equation as

$$
f(x, y)=h(y)-x y^{d-p-s}(y+1)^{s}=0
$$

its $y$-derivative is given by

$$
f_{y}(x, y)=h^{\prime}(y)+s x y^{d-p-s-1}(y+1)^{s-1} .
$$

Assume that $f$ and $f_{y}$ have a common zero $(a, b)$. So $f_{y}(a, b)=0$ which implies that $h^{\prime}(b)=-s a b^{d-p-s-1}(b+1)^{s-1}$. Also since $f(a, b)=0$, we have $s h(b)+b(b+1) h^{\prime}(b)=0$. But we see that $\operatorname{sh}(y)+y(y+1) h^{\prime}(y)=s \neq 0$ in $k[y]$, showing that $f$ and $f_{y}$ cannot have a common zero. Since the cover $\psi$ is non-trivial, it is branched only above $x=\infty$. Observe 
that the Equation 6.1 can be written in the following manner.

$$
\begin{aligned}
x^{-1} & =\frac{y^{d-p-s}(y+1)^{s}}{1+y^{d-s}(y+1)^{s}} ; \\
x^{-1} & =\frac{((y+1)-1)^{d-p-s}(y+1)^{s}}{1+((y+1)-1)^{d-s}(y+1)^{s}} ; \\
x^{-1} & =\frac{y^{-p}\left(y^{-1}+1\right)^{s}}{y^{-d}+\left(y^{-1}+1\right)^{s}} .
\end{aligned}
$$

Thus $v_{(y)}\left(x^{-1}\right)=d-p-s, v_{(y+1)}\left(x^{-1}\right)=s$, and $v_{\left(y^{-1}\right)}\left(x^{-1}\right)=p$. So there are exactly three points in $Y$ in the fibre of $\psi$ above $x=\infty$ with ramification indices $p, s$ and $d-p-s$. Let $\phi: \widetilde{Y} \rightarrow \mathbb{P}^{1}$ be the Galois closure of $\psi$ with Galois group $G$. Since 0 and -1 are the only roots of $y^{d-p-s}(y+1)^{s}$ and $h(0)=1=h(-1)$, by Lemma [2, Section 19, First Irreducibility Lemma], $f(x, y)$ is irreducible in $k(x)$ [y]. So $G$ is a transitive quasi $p$ subgroup of $S_{d}=$ $\operatorname{Sym}(z e r o s$ of $f$ ).

Proposition 6.3. Let $p$ be an odd prime, $a \geq 3$ be an integer, and let $d=$ ap. Let $\psi: Y \rightarrow \mathbb{P}^{1}$ be the degree-d cover given by affine Equation 6.1 Let $\phi: \widetilde{Y} \rightarrow \mathbb{P}^{1}$ be its Galois closure. Then $\phi$ is an $A_{d}$-Galois cover of $\mathbb{P}^{1}$ branched only at $\infty$, with inertia group $I=\langle\tau\rangle \rtimes\langle\beta\rangle$ above $\infty$ with $\tau$ a p-cycle, and $\beta$ of order l.c.m. $\left(\frac{p-1}{(p-1, d+1)}\right.$, l.c.m. $\left.(s, d-p-s)\right)$. Furthermore, it has upper jump $h / m=(d+1) /(p-1)$.

Proof. Let $x_{0}=x^{-1}$, and consider the local equation of $Y$ near a preimage of $x=\infty$. Then $I$ is the Galois group of the splitting field of $f(y)=x_{0}\left(1+y^{d-s}(y+1)^{s}\right)-y^{d-p-s}(y+$ $1)^{s}$ over $k\left(\left(x_{0}\right)\right)$. By Hensel's Lemma, $f(y)=g(y) h_{1}(y) h_{2}(y)$ in $k\left[\left[x_{0}\right]\right][y]$ with $g(y) \equiv$ $-1\left(\bmod x_{0}\right)$ of degree $p, h_{1}(y) \equiv y^{d-p-s}\left(\bmod x_{0}\right)$ of degree $d-p-s$, and $h_{2}(y) \equiv(y+$ $1)^{s}\left(\bmod x_{0}\right)$ of degree $s \leq p-1$. An argument similar to Proposition 4.3 shows that $h_{1}(y)$ is Eisenstein of degree $d-p-s$. Hence by Lemma 4.1. $\mathrm{Gal}\left(h_{1}, k\left(\left(x_{0}\right)\right)\right)=\mu_{d-p-s}$ and $p \nmid\left|\operatorname{Gal}\left(h_{2}(y), k\left(\left(x_{0}\right)\right)\right)\right|$. Also $p$ divides $|I|$ and $g$ has degree $p$. So $\operatorname{Gal}\left(g, k\left(\left(x_{0}\right)\right)\right)$ is a transitive subgroup of some $S_{p}=\operatorname{Sym}\left(\right.$ zeros of $g$ ). Hence $\operatorname{Gal}\left(g, k\left(\left(x_{0}\right)\right)\right) \leq S_{p} \cap G$. So the Sylow $p$-subgroup of $I$ is the same as the Sylow $p$-subgroup of $\mathrm{Gal}\left(g, k\left(\left(x_{0}\right)\right)\right)$, and it is generated by a $p$-cycle $\tau$. Without loss of generality, we may assume that $\tau=(1, \cdots, p)$.

By Proposition 2.1, the inertia group $I=\langle\tau\rangle \rtimes\langle\beta\rangle$, where $\beta=\theta^{i} \cdot \omega$ for some element $\theta$ in $S_{p}$ of order $(p-1)$, integer $0 \leq i \leq p-1$, and $\omega \in H=\operatorname{Sym}(\{p+1, \cdots, d\})$. Let $h$ be the conductor of the cover $\phi$, and let $\operatorname{ord}(\beta)=m$. Since there are exactly three points in $\psi^{-1}(\infty)$ with ramification indices $p, s$ and $d-p-s$, by Lemma 2.7 $\omega$ is of the form $\omega=\omega_{1} \cdot \omega_{2}$, where $\omega_{1}$ is an $s$-cycle and $\omega_{2}$ is a $(d-p-s)$-cycle disjoint from $\omega_{1}$. Now the Equations 2.1, 2.2 and 2.3 provide $\frac{h}{m}=\frac{d+1}{p-1}, \operatorname{ord}\left(\theta^{i}\right)=m^{\prime \prime}=\frac{p-1}{(p-1, d+1)}, m^{\prime}=\frac{\text { l.c.m. }(s, d-p-s)}{\left(m^{\prime \prime} \text {,.l.c.m. }(s, d-p-s) \text {. }\right.}$. Thus $m^{\prime \prime}$ equals $j$ and $\beta$ has order $m^{\prime} m^{\prime \prime}=1$.c.m. $\left(\frac{p-1}{(p-1, d+1)}\right.$, 1.c.m. $\left.(s, d-p-s)\right)$. As $\theta^{i}$ has order $m^{\prime \prime}=j, s \mid j$ and $(j, d-p-s)=1, \beta^{j}=\omega^{j}$ is a $(d-p-s)$-cycle. By Remark 6.2, we have $d-p-s>d / 2$. So the cycle $\beta^{j}$ fixes less than $d / 2$ points, and hence $G$ is primitive ([11, Remark 1.6]). So by Jordan's Theorem [10, Theorem 3.3.E], $G=A_{d}$.

Corollary 6.4. Let $p$ be an odd prime, $a \geq 3$ be an integer, and let $d=a p$. The $A_{d}$-Galois cover $\phi: \widetilde{Y} \rightarrow \mathbb{P}^{1}$ has the minimum possible upper jump among all the $A_{d}$-Galois covers of $\mathbb{P}^{1}$ étale away from $\infty$ for which the Sylow p-subgroup of the inertia group above $\infty$ is generated by a p-cycle.

Proof. As a consequence of the Riemann-Hurwitz formula (Equation 2.1), for any transitive subgroup $G$ of $S_{d}$, any $G$-Galois covers of $\mathbb{P}^{1}$ étale away from $\infty$ for which the Sylow 
$p$-subgroup of the inertia group above $\infty$ is generated by a $p$-cycle has upper jump $\geq \frac{d+1}{(p-1)}$. Now the result follows from Proposition 6.3.

\section{REFERENCES}

1. Abhyankar S. S., Coverings of algebraic curves, Amer. J. Math., 79, 825-856, 1957.

2. Abhyankar S. S., Galois theory on the line in nonzero characteristic, Bull. Amer. Math. Soc. (N.S.), 27(1), 68-133, 1992.

3. Abhyankar S. S., Resolution of singularities and modular Galois theory, Bull. Amer. Math. Soc. (N.S.), 38(2), 131-169, 2001.

4. Bouw I., and Pries R., Rigidity, reduction, and ramification, Math. Ann., 326(4), 803-824, 2003.

5. Bouw I., Covers of the affine line in positive characteristic with prescribed ramification, In WIN-Women in numbers, vol. 60 of Fields Inst. Commun., 193-200. Amer. Math. Soc., Providence, RI, 2011.

6. Harbater D., Formal patching and adding branch points, American Journal of Mathematics, 115, 487-508, 1993.

7. Harbater D., Abhyankar's Conjecture and embedding problems, J. Reine Angew. Math. 559, 1-24, 2003.

8. Harbater D., Obus A., Pries R., Stevenson, K., Abhyankar's Conjectures in Galois Theory: Current Status and Future Directions, Bull. Amer. Math. Soc. (N.S.) 55, no. 2, 239-287, 2018.

9. Harbater D., Embedding problems with local conditions, Israel J. Math., 118, 317-355, 2000.

10. Dixon J. D., Mortimer B., Permutation Groups, Springer-Verlag, NY-Heidelberg-Berlin, 1996.

11. Jones, G. A., Primitive permutation groups containing a cycle, Bull. Aust. Math. Soc., 89, no. 1, 159-165, 2014.

12. Kumar M., Compositum of Wildly Ramified Extensions, J. Pure Appl. Algebra 218, no. 8, 1528-1536, 2014.

13. Kumar M., Killing Wild ramification, Israel J. Math. 199, no. 1, 421-431, 2014.

14. Muskat J., Pries R., Alternating group covers of the affine line, Israel J. Math., 187, 117-139, 2012.

15. Obus A., Toward Abhyankar's Inertia Conjecture for $P S L_{2}(l)$, Geometric and differential Galois theories, 195-206, Sémin. Congr., 27, Soc. Math. France, Paris, 2013.

16. Pries R., Conductors of wildly ramified covers, III, Pacific J. Math, no.1, 221, 163-182, 2003.

17. Raynaud M., Revêtements de la droite affine en caractéristique $p>0$ et conjecture d'Abhyankar, Invent. Math., 116(1-3), 425-462, 1994.

18. Revêtements étales et groupe fondamental (French), Séminaire de Géométrie Algébrique du Bois Marie 1960-1961 (SGA 1). Dirigé par Alexandre Grothendieck. Augmenté de deux exposés de Michéle Raynaud. Lecture Notes in Mathematics, Vol. 224. Springer-Verlag, Berlin-New York, 1971.

19. Serre J. P., Local fields, vol. 67, Graduate Texts in Mathematics. Springer-Verlag, New York, 1979. Translated from the French by Marvin Jay Greenberg.

20. Serre J. P., Construction de revêtements étales de la droite affine en caractéristique p, C. R. Acad. Sci. Paris Sér. I Math., 311(6), 341-346, 1990.

Statistics and Mathematics Unit, Indian Statistical Institute, Bangalore Center, Bangalore 560059.

E-mail address: soumyadip_rs@isibang.ac.in

Statistics and Mathematics Unit, Indian Statistical Institute, Bangalore Center, Bangalore 560059.

E-mail address: manish@isibang.ac.in 